\title{
Food and beverage cues in children's television programmes: the influence of programme genre
}

\author{
Paul Scully ${ }^{1}$, Orlaith Reid' ${ }^{1}$, Alan Macken ${ }^{1,2,3}$, Mark Healy ${ }^{4}$, Jean Saunders ${ }^{4}$, \\ Des Leddin ${ }^{3,5}$, Walter Cullen $^{3}$, Colum Dunne ${ }^{\prime}$ and Clodagh $S \mathrm{O}^{\prime}$ Gorman ${ }^{1,2,3,5, *}$ \\ ${ }^{1}$ The Children's Ark, University Hospital Limerick, Limerick, Republic of Ireland: ${ }^{2}$ National Children's Research \\ Centre, Dublin, Republic of Ireland: ${ }^{3}$ Centre for Interventions in Infection, Inflammation \& Immunity (4i), Graduate \\ Entry Medical School, Limerick, Republic of Ireland: ${ }^{4} \mathrm{C}$-Star, University of Limerick, Limerick, Republic of Ireland: \\ ${ }^{5}$ Dalhousie University, Halifax, Nova Scotia, Canada
}

Submitted 17 June 2014: Final revision received 31 March 2015: Accepted 15 April 2015: First published online 17 June 2015

\begin{abstract}
Objective: The link between childhood obesity and both television viewing and television advertising have previously been examined. We sought to investigate the frequency and type of food and beverage placements in children-specific television broadcasts and, in particular, differences between programme genres. Method: Content of five weekdays of children-specific television broadcasting on both UK (BBC) and Irish (RTE) television channels was summarized. Food and beverage placements were coded based on type of product, product placement, product use and characters involved. A comparison was made between different programme genres: animated, cartoon, child-specific, film, quiz, tween and young persons' programming.

Results: A total of $1155(\mathrm{BBC}=450 ; \mathrm{RTE}=705)$ cues were recorded giving a cue every $4.2 \mathrm{~min}$, an average of $12.3 \mathrm{~s} / \mathrm{cue}$. The genre with most cues recorded was cartoon programming $(30 \cdot 8 \%)$. For the majority of genres, cues related to sweet snacks (range 1.8-23.3\%) and sweets/candy (range 3.6-25.8\%) featured highly. Fast-food (18.0\%) and sugar-sweetened beverage (42.3\%) cues were observed in a high proportion of tween programming. Celebratory/social motivation factors (range 10-40\%) were most common across all genres while there were low proportions of cues based on reward, punishment or healthrelated motivating factors.

Conclusions: The study provides evidence for the prominence of energy-dense/ nutrient-poor foods and beverages in children's programming. Of particular interest is the high prevalence of fast-food and sugar-sweetened beverage cues associated with tween programming. These results further emphasize the need for programme makers to provide a healthier image of foods and beverages in children's television.
\end{abstract}

In the developed world, obesity is now the most common disease of childhood and adolescence and its prevalence continues to rise ${ }^{(1)}$. Some contributing factors include more sedentary time and less active time, larger portion sizes and poorer choices in foods consumed ${ }^{(2)}$. A healthy balanced diet provides recommended amounts of nutrients and other food components to promote normal growth and development, reduce chronic disease risk, and foster appropriate energy balance and a healthy weight trajectory. However, the current eating regimes of children and adolescents differ markedly from recommended patterns and as a result represent a health risk ${ }^{(3)}$.

Previous studies have found a strong dose-response relationship between the prevalence of overweight in children and hours of television viewed ${ }^{(4,5)}$. The American Academy of Pediatrics recommends that children over the age of 2 years should watch no more than $2 \mathrm{~h}$ of non-educational television per day ${ }^{(6)}$. Recent evidence suggests that children aged 6-11 years watch as much as $24.5 \mathrm{~h} /$ week, $10 \mathrm{~h}$ more than the recommended watching ${ }^{(7)}$. Within the UK, data released through the Ofcom show that the average number of minutes of television people aged 4 years and over watch each day is $3 \mathrm{~h}$ and $52 \mathrm{~min}^{(8)}$. Additionally, they also show that children aged 6-11 years watch a total of $2 \mathrm{~h}$ and $55 \mathrm{~min}$ of television daily with $1 \mathrm{~h}$ and $51 \mathrm{~min}$ being live television, while for children between aged 11-15 years the total television watched is $2 \mathrm{~h}$ and 57 min with $1 \mathrm{~h}$ and 32 min of live television ${ }^{(8)}$. 
Television viewing is associated with greater periods of inactivity and exposure to food advertising and products $^{(9,10)}$. More specifically, it has been shown that over $99 \%$ of food-product advertisements viewed by children are for foods high in fat, sugar or sodium ${ }^{(11)}$. Furthermore, compared with lean children, obese children may be more easily influenced by food advertisements and they more readily recognize specific food-related commercials and consume more food following exposure ${ }^{(12,13)}$.

Children, particularly younger children, are more susceptible and have lower cognitive defences against television marketing $^{(14,15)}$. From age 2 to 11 years, children develop consumption motives and values as they are exposed to commercial activities and develop knowledge about advertising, products, brands, pricing and shopping, and begin to develop strategies for purchase requests and negotiation ${ }^{(3)}$. Strong evidence exists that television and television advertising influence the food and beverage preferences, purchase requests, beliefs and short-term consumption of children aged 2-11 years, but data for children aged $12-18$ years are insufficient ${ }^{(3)}$. Additionally, there is moderate evidence that television advertising influences the usual dietary intake of younger children and weak evidence that it influences the usual dietary intake of older children or teens ${ }^{(3)}$. Research also shows that from 6 years onwards, children start to discriminate and develop an understanding of food types that are good for them ${ }^{(16)}$. However, there may be other factors that influence the preference for and liking of food ${ }^{(17)}$. For example, children appreciate aesthetic features and play features of food. Boys especially like foods with strange colours and shapes, while girls appreciate pretty colours and general aesthetic appeal ${ }^{(18)}$. More recent studies investigating children's discourses and practices using multiple qualitative techniques show different appeals and social influences behind children's food preferences, including sensory, fun and parental influencers ${ }^{(19)}$. As a consequence such studies have shown that age, gender and social status influence food preference and choice. As children-specific programming is produced for and aimed at different age groups, it is interesting to speculate on differences that may exist within such programming in the portrayal of foods and beverages.

Therefore the aim of the present study was to describe and examine the portrayal, motivating factors and outcomes of food and beverage placements within different genres of children's television. The study was conducted on public broadcast channels as these are non-profit in nature with a mission statement to offer positive and trusted voices in broadcasting as well as informing, educating and empowering audiences ${ }^{(20,21)}$.

\section{Method}

\section{Programme recording}

Content analysis of UK and Irish children's television programmes was conducted, including five weekdays of programmes broadcast during periods of continuous broadcasts of children's-specific television on BBC and RTE. Programmes were recorded in July 2010 and October 2010 , totalling $82.5 \mathrm{~h}$. The programmes' country of origin and year of production were noted. The programmes were grouped on the basis of genre into eight groups: animated, cartoon, child-specific, film, quiz, tween, young person and music. The genre types were obtained from information obtained from production and television channel notes for each of the individual programmes. Where there was ambiguity or disagreement, consensus was achieved with the principal supervisor (C.S.O.). The child-specific genre represented those programmes that were produced for those less than 10 years of age. The tween genre represented programmes, largely comedic in nature, that were aimed at teenage viewers. The young person grouping represented those programmes aimed at a combined child and teenage age group.

\section{Data coding}

\section{Food/beverage placement}

Recorded programmes were viewed individually by two investigators (P.S., O.R.). A food or beverage cue was defined as a product being displayed within a foodspecific context with potential to be consumed. Cues were noted for food type but not further coded if depicted in a non-food context (e.g. a programme called 'Toast'; a character called 'Cowardy Custard'). Multiple cues within a scene were each individually recorded. Cues were coded by type of product and as healthy or energy-dense/nutrient-poor (EDNP; based on the tip of the food pyramid, where such foods are not recommended as they are not considered essential ${ }^{(22)}$ ). Healthy foods included breads/grains, cereals, meats, dairy, fruit, vegetables, fish, sandwiches; EDNP foods included fastfood/convenience meals, pastries, savoury snacks, sweet snacks/bars, ice cream and candy. Beverages were coded and grouped as water, juices, tea/coffee, sugar-sweetened or unspecified.

\section{Food/beverage context}

Cues were coded as presented to the child visually, verbally or both. Background cues were not coded unless specifically labelled. Cue duration was recorded. Cues reappearing in the same scene were not re-coded. Cue context was recorded: ingestion acts, whether the cue was offered and accepted, whether it was part of a meal, or within a home or school setting.

\section{Characters}

Characters associated with the cue were recorded: human, animated human or animated characters. If human, estimates of age, gender, ethnicity and weight status were documented. 


\section{Cue motivation and outcome}

Motives related to the cue were recorded under several classifications and multiple motives were recorded under the same cue if necessary. Motives included social, hunger/thirst, upset, excitement, weight-related, reward, punishment and health-related. As only a number of these classifications were observed at a statistically significant level, they were subsequently classified into positive motivations (social, excitement, reward), negative motivations (upset, punishment), health-related motivations (hunger/thirst, weight, healthy living) and miscellaneous motives. The outcome of the cue was coded as being positive, negative or neutral connotation. A positive outcome included enjoyment, winning a race or prize, aid to other people or feeling better following the cue. Negative outcomes included pain or personal harm, disgust or physical grimacing or crying, excessive consumption, vomiting or harm to other people.

\section{Statistical analysis}

Data collection was piloted and reviewed after $1 \mathrm{~d}$ of sample programming where ambiguity in cues was discussed. Pilot data were excluded from the final analysis. Inter-rater reliability using pilot data was reviewed at hourly intervals until $\kappa$ reliabilities of 0.80 were achieved for each variable. Where there was any inter-coder variability or uncertainty, the programme was re-viewed, discussed and consensus achieved with a principal supervisor (C.S.O.G.). Further analysis was performed using the statistical software package IBM SPSS Statistics 20. Food and beverage cues across each genre were compared using $\chi^{2}$ testing. Means of cue duration were compared using an independent $t$ test. Statistical significance was set at $P<0 \cdot 05$.

\section{Results}

\section{Recording statistics}

A total of $1155(\mathrm{RTE}=705 ; \mathrm{BBC}=450)$ food and beverage cues were recorded over the $82.5 \mathrm{~h}$ recording period, giving a new cue every $4.2 \mathrm{~min}$. The total recorded time involving food and beverage cues was $14189 \mathrm{~s}(3.94 \mathrm{~h})$, an average of $12.3 \mathrm{~s} / \mathrm{cue}$. Food and beverage cues amounted to $4.8 \%$ of the total recorded period. The recording period included fifty-five individual programmes, produced in nine countries. Programmes produced in the USA were most common (39.5\%) followed by the UK (23.2\%) and Ireland (15.9\%). The year of production ranged from 1955 to 2010 , with $66 \%$ of recordings produced between 2004 and 2010.

The genre with most cues recorded was cartoon programming $(30 \cdot 8 \%)$, followed by animated programming $(17 \cdot 8 \%)$, tween programming (13.2\%) and feature films $(12 \cdot 6 \%)$. Based on the low number of cues associated with music programming, this genre was omitted from the final analysis.

\section{Food and beverage types}

Fourteen specific food types were depicted during the recording period (Table 1). When specific foods were analysed across the programme genres, sweet snacks and sweets/candy were within the three most common depictions for the majority (Fig. 1). Fruit-related cues also featured frequently across most genres. Tween programming was the only genre that most commonly depicted fast-food/convenience meals (18.0\%) and savoury snacks (11.0\%). Of the specific food cues, EDNP food products accounted for $68.2 \%$ of animated programmes, $52.0 \%$ of cartoon programmes, $42.4 \%$ of children-specific programmes, $29.6 \%$ of feature films, $15.9 \%$ of quiz programmes, $54.0 \%$ of tween programmes and $34.4 \%$ of young persons' programmes (Fig. 2).

Five specific beverage types were depicted during the recording period (Table 1). Across the majority of genres, unspecified beverage cues were most commonly recorded. With regard to specific beverage cues, sugarsweetened beverages were seen commonly in tween programming $(42.3 \%)$ and not at all in children-specific programming or feature films (Fig. 1).

\section{Cue usage}

There were varying proportions of usage related to each genre (Table 2). For cue type, cartoon programming was associated with a lower proportion of visual type cues (26.7\%) with quiz (50.0\%) and young persons' (49.0\%) programming showing increased proportions of verbal type cues. For each genre, the majority of cues were within the programme foreground. Quiz programming showed the greatest proportion of cues with a non-food context at $48.5 \%$. Films (55.2\%) and tween (43.4\%) programming most commonly involved an act of ingestion, with approximately a quarter of cues involving consumption acts for the other genres. When location was assessed, a larger proportion of tween programming cues took place within the home $(51 \cdot 0 \%)$.

\section{Characters}

Throughout the various genres, male characters were most prominent (range 20.0-94.5\%; Table 2). There were various proportions of character ages between the genres but of note was the increased proportion of teenage characters $(81.5 \%)$ in tween programming and the high proportion of adults in quiz (88.6\%) and young persons' (78.7\%) programmes. Similar proportions of overweight characters (range 5.8-9.7\%) were noted through most genres apart from an increased proportion in young persons' programming (29.8\%) and no overweight characters within child-specific programmes.

\section{Outcome and motivation}

For each genre, apart from quiz programming, the most common motivating factors were celebratory/social and 
hunger/thirst (Table 3). There were low proportions of cues based on reward or punishment motivating factors (less than 5\% for all genres, except quiz programming). Health-motivated cues were seen only in cartoon programming (1.4\%), quiz programming (17.1\%) and young persons' programming (5.5\%). Positive motivations overall were most commonly seen with children-specific (40.5\%) and animated (38.5\%) programmes, while negative motivations overall were most commonly seen in films (4.4\%) and tween ( $2.7 \%)$ programmes. There was similar cue outcome for the each of the genre types for positive (range 27.4-48.4\%), negative (range 0-24.7\%) and neutral outcomes (range $40.5-56.2 \%$ ). Of note, there were no negative outcome cues associated with children-specific programming.

\section{Discussion}

In the present study, we examined the variation in food and beverage cues between a number of different programme genres, following a previous study by our group which showed that within children's programming sweet snacks were most commonly seen in Irish and UK programming ${ }^{(23)}$. To date there has been a wide range of studies undertaken assessing food and beverage advertisements broadcast during children-specific programming but there have been few studies examining in detail the portrayal of foods and beverages within the actual programming itself.

We found a high percentage of cues for EDNP foods in the present study, with the proportion being greater than half of all food cues for the majority of genres. Of particular interest was the high proportion of fast foods and convenience foods seen within tween programming and the high proportion of sweet snacks and sweets/candy-related cues in animated, cartoon and children-specific genre programmes. This difference in the type of EDNP food cue depicted is largely due to the nature of the programme types but has the potential to influence food preferences in differing age groups. Fruit- and vegetable-related cues were evenly distributed across most genres (about $10 \%$ ), with children-specific

Table 1 Comparison of food and beverage cues by programme genre in a content analysis of five weekdays of children-specific television broadcasting on both UK (BBC) and Irish (RTE) television channels (82.5 h in total), July 2010 and October 2010

\begin{tabular}{|c|c|c|c|c|c|c|c|c|c|c|c|c|c|c|c|c|}
\hline & \multicolumn{2}{|c|}{$\begin{array}{c}\text { Animated } \\
(n \text { 206) }\end{array}$} & \multicolumn{2}{|c|}{$\begin{array}{l}\text { Cartoon } \\
(n 356)\end{array}$} & \multicolumn{2}{|c|}{$\begin{array}{l}\text { Child } \\
(n 40)\end{array}$} & \multicolumn{2}{|c|}{$\begin{array}{c}\text { Film } \\
(n 146)\end{array}$} & \multicolumn{2}{|c|}{$\begin{array}{c}\text { Quiz } \\
(n 137)\end{array}$} & \multicolumn{2}{|c|}{$\begin{array}{l}\text { Tween } \\
(n 152)\end{array}$} & \multicolumn{2}{|c|}{$\begin{array}{l}\text { Young person } \\
\quad(n 114)\end{array}$} & \multirow[b]{2}{*}{$\chi^{2} /$ ANOVA } & \multirow[b]{2}{*}{$P$} \\
\hline & $n$ & $\%$ & $n$ & $\%$ & $n$ & $\%$ & $n$ & $\%$ & $n$ & $\%$ & $n$ & $\%$ & $n$ & $\%$ & & \\
\hline Foods & \multicolumn{2}{|c|}{ (n 159) } & \multicolumn{2}{|c|}{ (n 290) } & \multicolumn{2}{|c|}{ (n 33) } & \multicolumn{2}{|c|}{$(n 90)$} & \multicolumn{2}{|c|}{$(n$ 123) } & \multicolumn{2}{|c|}{ (n 100) } & \multicolumn{2}{|c|}{ (n 79) } & & \\
\hline Unspecified & 29 & $18 \cdot 2$ & 43 & $14 \cdot 8$ & & & 24 & $26 \cdot 7$ & 24 & $21 \cdot 4$ & 12 & $12 \cdot 0$ & 12 & $15 \cdot 2$ & $18 \cdot 2^{\star}$ & 0.01 \\
\hline Breads/grains & 2 & $1 \cdot 3$ & 15 & $5 \cdot 2$ & 4 & $12 \cdot 1$ & 7 & $7 \cdot 8$ & 24 & 21.4 & 1 & 1.0 & 2 & 2.5 & $59 \cdot 5^{\star \star \star}$ & 0.00 \\
\hline Cereals & 2 & 1.3 & 8 & 2.8 & 5 & $15 \cdot 2$ & 4 & 4.4 & 2 & $1 \cdot 8$ & & 0 & 5 & $6 \cdot 3$ & $25 \cdot 9^{\star \star}$ & 0.001 \\
\hline Meats & \multicolumn{2}{|r|}{0} & 12 & $4 \cdot 1$ & \multicolumn{2}{|c|}{0} & 8 & 8.9 & 2 & $1 \cdot 8$ & 10 & $10 \cdot 0$ & 7 & 8.9 & $25 \cdot 7^{\star \star}$ & 0.001 \\
\hline Dairy & 1 & 0.6 & 7 & 2.4 & \multicolumn{2}{|c|}{0} & 1 & $1 \cdot 1$ & 10 & 8.9 & 3 & 3.0 & 15 & $19 \cdot 0$ & $59 \cdot 5^{\star \star \star}$ & 0.00 \\
\hline Condiments & \multicolumn{2}{|r|}{0} & 5 & 2.4 & \multicolumn{2}{|c|}{0} & 5 & $5 \cdot 6$ & 10 & 8.9 & 4 & $4 \cdot 0$ & 2 & 2.5 & $108 \cdot 0^{\star \star \star}$ & 0.00 \\
\hline Fruit & 21 & $13 \cdot 2$ & 26 & $9 \cdot 0$ & \multicolumn{2}{|c|}{$27 \cdot 3$} & 7 & $7 \cdot 8$ & 12 & $10 \cdot 7$ & 14 & $14 \cdot 0$ & 8 & $10 \cdot 1$ & $13 \cdot 0$ & 0.07 \\
\hline Vegetables & 9 & 5.7 & 32 & 11.0 & 1 & 3.0 & 9 & $10 \cdot 0$ & 4 & 3.6 & 4 & 4.0 & 5 & $6 \cdot 3$ & $12 \cdot 6$ & 0.08 \\
\hline Fish & 6 & 3.8 & 11 & 3.8 & \multicolumn{2}{|c|}{0} & 4 & $4 \cdot 4$ & \multicolumn{2}{|r|}{0} & 4 & $4 \cdot 0$ & & 0 & $9 \cdot 3$ & 0.23 \\
\hline Sandwiches & & 0 & & 0 & & & & 0 & & 0 & & 0 & & 0 & & \\
\hline Fast foods & 1 & 0.6 & 11 & 3.8 & & & 1 & $1 \cdot 1$ & & 0 & 18 & $18 \cdot 0$ & 1 & 1.3 & $70 \cdot 4^{\star \star \star}$ & 0.00 \\
\hline Breakfast pastries & & 0 & 6 & $2 \cdot 1$ & & & 1 & $1 \cdot 1$ & 4 & 3.6 & 1 & 1.0 & & 0 & 8.92 & 0.26 \\
\hline Savoury snacks & 5 & $3 \cdot 1$ & 11 & $3 \cdot 8$ & & & 3 & $3 \cdot 3$ & 2 & $1 \cdot 8$ & 11 & 11.0 & 5 & 6.3 & $16 \cdot 0^{\star}$ & 0.03 \\
\hline Sweet snacks & 37 & $23 \cdot 3$ & 51 & $17 \cdot 6$ & 7 & $21 \cdot 2$ & 4 & 4.4 & 2 & 3.6 & 4 & 4.0 & 10 & $12 \cdot 7$ & $47 \cdot 2^{\star \star \star}$ & 0.00 \\
\hline Ice cream & 5 & 3.1 & 17 & 5.9 & 3 & $9 \cdot 1$ & 3 & $3 \cdot 3$ & & 0 & 5 & $5 \cdot 0$ & 1 & $1 \cdot 3$ & $12 \cdot 0$ & 0.10 \\
\hline Sweets/candy & 41 & $25 \cdot 8$ & 29 & $10 \cdot 0$ & 4 & $12 \cdot 1$ & 7 & $7 \cdot 8$ & & 0 & 8 & $8 \cdot 0$ & 6 & $7 \cdot 6$ & $43 \cdot 7^{\star \star \star}$ & 0.00 \\
\hline Others & & 0 & 6 & $2 \cdot 1$ & & & 2 & $2 \cdot 2$ & 12 & $10 \cdot 0$ & 1 & 1.0 & & 0 & $40 \cdot 4^{\star \star \star}$ & 0.00 \\
\hline EDNP (\%) & & $8 \cdot 2$ & & $2 \cdot 0$ & & & & $9 \cdot 6$ & & $5 \cdot 9$ & & $4 \cdot 0$ & & $4 \cdot 4$ & & \\
\hline Beverages & & 29) & & 40) & & & & $55)$ & & 24) & & 52) & & 19) & & \\
\hline Unspecified & 19 & 65.5 & 21 & $52 \cdot 5$ & 2 & $66 \cdot 7$ & 12 & $21 \cdot 8$ & 6 & $25 \cdot 0$ & 15 & 28.8 & 3 & $15 \cdot 8$ & $28 \cdot 3^{\star \star \star}$ & 0.00 \\
\hline Water & & 0 & 3 & 7.5 & & & 2 & 3.6 & & 0 & 3 & 5.8 & 2 & 10.5 & $24 \cdot 3^{\star \star}$ & 0.001 \\
\hline Milk & & 0 & 3 & $7 \cdot 5$ & & & 3 & $5 \cdot 5$ & 2 & $8 \cdot 3$ & 1 & 1.9 & 8 & $42 \cdot 1$ & $37 \cdot 6^{\star \star \star}$ & 0.00 \\
\hline Juice & 2 & 6.9 & 3 & 7.5 & 1 & $33 \cdot 3$ & 3 & 5.5 & & 0 & 3 & $5 \cdot 8$ & & 0 & $7 \cdot 61$ & 0.37 \\
\hline Tea/coffee & 3 & $10 \cdot 3$ & 4 & $10 \cdot 0$ & & & 5 & $9 \cdot 1$ & 12 & $50 \cdot 0$ & 3 & $5 \cdot 8$ & 3 & $15 \cdot 8$ & $32 \cdot 4^{\star \star \star}$ & 0.00 \\
\hline Sugar-sweetened & 2 & 6.9 & 3 & $7 \cdot 5$ & & & & 0 & 2 & $8 \cdot 3$ & 22 & $42 \cdot 3$ & & 0 & $53 \cdot 6^{\star \star \star}$ & 0.00 \\
\hline Other & 3 & $10 \cdot 3$ & 3 & $7 \cdot 5$ & & & 30 & $54 \cdot 0$ & 2 & $8 \cdot 3$ & 5 & $9 \cdot 6$ & 3 & $15 \cdot 8$ & $52 \cdot 1^{\star \star \star}$ & 0.00 \\
\hline & Mean & SE & Mean & SE & Mean & SE & Mean & SE & Mean & SE & Mean & SE & Mean & SE & $P$ & \\
\hline Cue duration (s) & & & & & & & & & & & & & & & & \\
\hline Overall & $20 \cdot 8$ & $2 \cdot 5$ & $13 \cdot 2$ & $1 \cdot 3$ & $23 \cdot 3$ & $4 \cdot 0$ & $14 \cdot 8$ & 3.0 & $9 \cdot 2$ & $3 \cdot 2$ & $3 \cdot 1 \dagger$ & 0.5 & $14 \cdot 6$ & $2 \cdot 8$ & 0.00†† & \\
\hline Healthy & $20 \cdot 6$ & $4 \cdot 3$ & $16 \cdot 4$ & 2.4 & $25 \cdot 6$ & $5 \cdot 6$ & 10.6 & 3.4 & $13 \cdot 4$ & 4.9 & $2 \cdot 7 \dagger$ & 0.7 & $10 \cdot 2$ & 2.5 & $0.034 \dagger$ & \\
\hline EDNP & $18 \cdot 2$ & 3.0 & 11.4 & 1.6 & $14 \cdot 8$ & $5 \cdot 3$ & 9.5 & $5 \cdot 7$ & $0.7 \dagger$ & 0.1 & $3.4 \dagger$ & 0.8 & $12 \cdot 3$ & $4 \cdot 8$ & $0.001 \dagger$ & \\
\hline
\end{tabular}

ENDP, energy-dense/nutrient-poor.

Values are given are frequencies and total relative percentages. Numerical data are expressed as means with their standard errors.

Level of significance (using $\chi^{2}$ test for categorical values): ${ }^{\star} P<0.05,{ }^{\star} P<0.01,{ }^{\star \star \star} P<0.001$.

Level of significance (using $t$ test/ANOVA for numerical values): $\dagger P<0.05, \dagger+P<0.01, \dagger+\uparrow P<0.001$. 
(a)

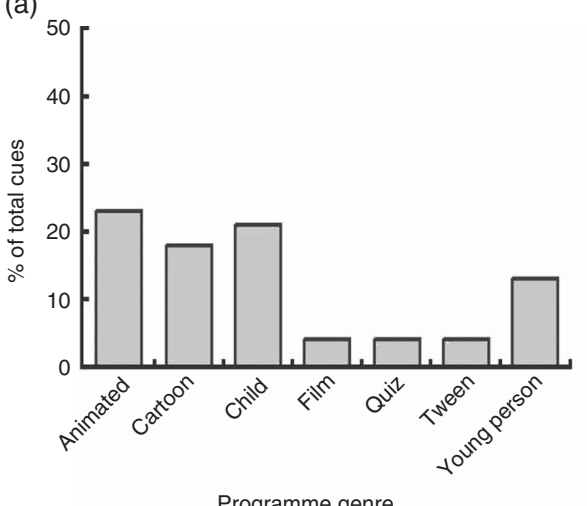

(c)

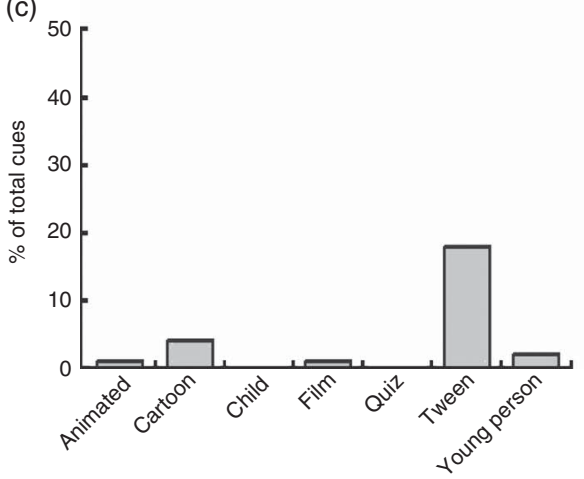

Programme genre (b)

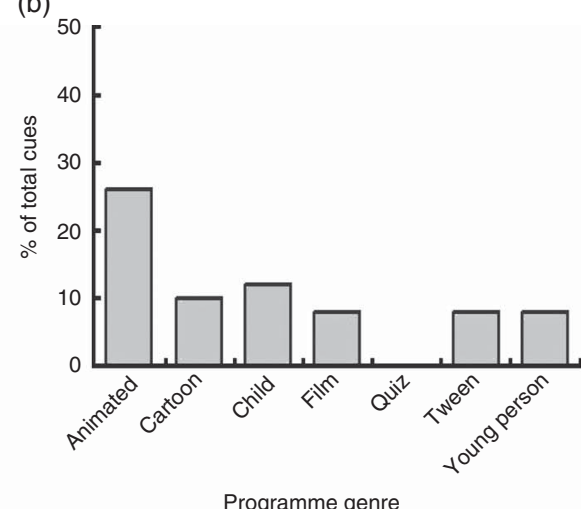

(d)

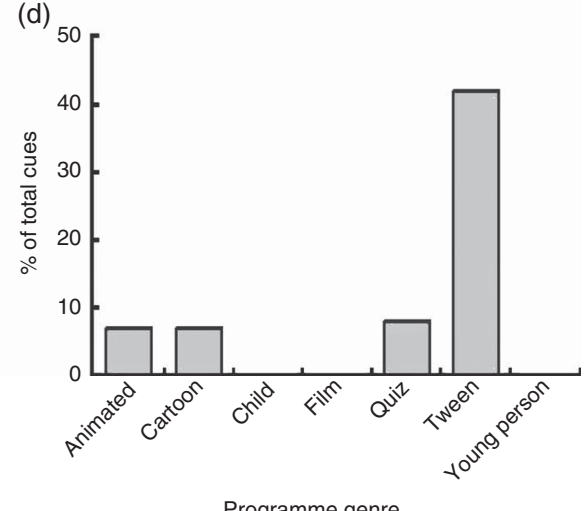

Fig. 1 Percentage of total cues $(n$ 1155) for (a) sweet snacks, (b) sweets/candy, (c) fast foods and (d) sugar-sweetened beverages, by programme genre, in a content analysis of five weekdays of children-specific television broadcasting on both UK (BBC) and Irish (RTE) television channels $(82.5 \mathrm{~h}$ in total), July 2010 and October 2010

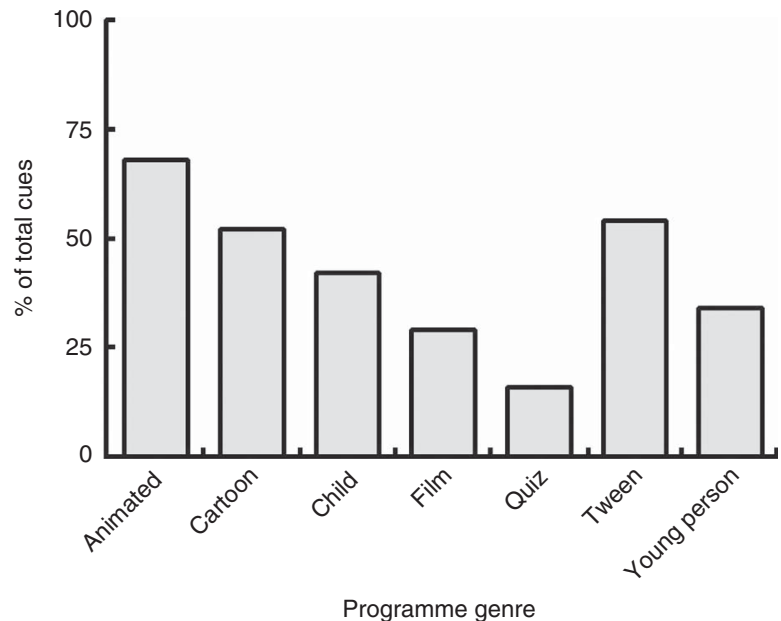

Fig. 2 Percentage of total cues (n 1155) for energy-dense/ nutrient-poor foods, by programme genre, in a content analysis of five weekdays of children-specific television broadcasting on both UK (BBC) and Irish (RTE) television channels $(82.5 \mathrm{~h}$ in total), July 2010 and October 2010

programming showing a high proportion of fruit-related cues at $27 \%$. Thus, these results further serve to highlight the high proportion of EDNP foods depicted while additionally showing that this is independent of programme genre. The high proportion of EDNP food depictions within advertising has been well documented, with studies showing that $97 \cdot 8 \%$ of food-product advertisements viewed by children aged 2-11 years were for products high in fat, sugar or sodium, with poor nutritional value but of high energy content ${ }^{(11)}$. More recently, in a study of food cues in television programmes for children under 5 years old, there was almost twice the total airtime for unhealthy foods (simple sugars, fats and simple carbohydrates) compared with healthy foods within children's television programmes, and characters in the programmes valued and endorsed unhealthy foods more often than healthy foods ${ }^{(24)}$. A complementary study showed that television viewing was inversely associated with intake of fruit and vegetables among adolescents and that these associations may be a result of the replacement of fruits and vegetables in youths' diets by foods highly advertised on television ${ }^{(25)}$. The results from our current study further serve to highlight these findings and additionally show that such features of high proportions of EDNP foods and limited fruit and vegetable portrayals occur across a variety of different genres of children's programming.

We also examined beverage cues within children's programming genres. There was a high proportion of 
Table 2 Comparison of type, usage, location and characters in food and beverage cues, by programme genre, in a content analysis of five weekdays of children-specific television broadcasting on both UK (BBC) and Irish (RTE) television channels (82.5 h in total), July 2010 and October 2010

\begin{tabular}{|c|c|c|c|c|c|c|c|c|c|c|c|c|c|c|c|c|}
\hline & \multicolumn{2}{|c|}{$\begin{array}{c}\text { Animated } \\
(n \text { 206) }\end{array}$} & \multicolumn{2}{|c|}{$\begin{array}{l}\text { Cartoon } \\
(n \text { 356) }\end{array}$} & \multicolumn{2}{|r|}{$\begin{array}{l}\text { Child } \\
(n \text { 40) }\end{array}$} & \multicolumn{2}{|c|}{$\begin{array}{c}\text { Film } \\
(n \text { 146) }\end{array}$} & \multicolumn{2}{|c|}{$\begin{array}{l}\text { Quiz } \\
\text { (n 137) }\end{array}$} & \multicolumn{2}{|c|}{$\begin{array}{l}\text { Tween } \\
(n \text { 152) }\end{array}$} & \multicolumn{2}{|c|}{$\begin{array}{l}\text { Young person } \\
\qquad(n 114)\end{array}$} & \multirow[b]{2}{*}{$\chi^{2}$} & \multirow[b]{2}{*}{$P$} \\
\hline & $n$ & $\%$ & $n$ & $\%$ & $n$ & $\%$ & $n$ & $\%$ & $n$ & $\%$ & $n$ & $\%$ & $n$ & $\%$ & & \\
\hline \multicolumn{17}{|l|}{ Cue type } \\
\hline Visual & 92 & $49 \cdot 2$ & 88 & $26 \cdot 7$ & 19 & $52 \cdot 8$ & 60 & 41.4 & 28 & $20 \cdot 6$ & 68 & 44.7 & 25 & 25.5 & $56 \cdot 8^{\star \star \star}$ & 0.00 \\
\hline Verbal & 15 & 8.0 & 83 & $25 \cdot 2$ & 3 & 8.3 & 31 & 21.4 & 68 & $50 \cdot 0$ & 29 & $19 \cdot 1$ & 48 & 49.0 & $111^{\star \star \star}$ & 0.00 \\
\hline Combined & 80 & $42 \cdot 2$ & 158 & 48.0 & 14 & 38.9 & 54 & $37 \cdot 2$ & 40 & 29.4 & 55 & $36 \cdot 2$ & 25 & 25.5 & $25 \cdot 9^{\star \star}$ & 0.001 \\
\hline Foreground & 165 & 88.0 & 325 & 99.0 & 36 & 100.0 & 139 & 95.0 & 134 & 98.0 & 142 & 93.0 & 93 & 94.9 & $39 \cdot 9^{\star \star \star}$ & 0.00 \\
\hline Non-food context & 15 & 8.0 & 40 & $12 \cdot 2$ & 5 & 13.9 & 14 & 9.7 & 66 & 48.5 & 6 & 3.9 & 28 & 28.6 & $160^{\star \star \star}$ & 0.00 \\
\hline \multicolumn{17}{|l|}{ Cue usage } \\
\hline Offered & 37 & 21.3 & 56 & $17 \cdot 4$ & 9 & 22.5 & 31 & 21.4 & 5 & 2.9 & 28 & $18 \cdot 4$ & 12 & 14.8 & $31 \cdot 7^{\star \star \star}$ & 0.00 \\
\hline Accepted & 33 & 19.0 & 41 & $12 \cdot 8$ & \multicolumn{2}{|c|}{0} & 23 & $15 \cdot 9$ & 2 & 1.5 & 18 & 11.8 & 8 & 9.9 & $29 \cdot 7^{\star \star \star}$ & 0.00 \\
\hline Ingested & 48 & $27 \cdot 6$ & 96 & 29.9 & \multicolumn{2}{|c|}{$10 \quad 32 \cdot 3$} & 80 & $55 \cdot 2$ & 16 & 11.8 & 66 & 43.4 & 22 & $27 \cdot 2$ & $74 \cdot 9^{\star \star \star}$ & 0.00 \\
\hline \multicolumn{17}{|l|}{ Location } \\
\hline Home & 48 & $28 \cdot 1$ & 85 & 29.4 & \multirow[t]{2}{*}{4} & \multirow{2}{*}{$0^{12 \cdot 9}$} & 51 & 38.9 & 26 & 37.1 & \multirow{2}{*}{\multicolumn{2}{|c|}{$\begin{array}{rr}74 & 51.0 \\
8 & 5.5\end{array}$}} & \multirow{2}{*}{\multicolumn{2}{|c|}{$\begin{array}{l}4 \cdot 1 \\
2 \cdot 7\end{array}$}} & $61 \cdot 8^{\star \star \star}$ & \multirow{2}{*}{$\begin{array}{l}0.00 \\
0.005\end{array}$} \\
\hline School & & 0 & 26 & 9.0 & & & 8 & $6 \cdot 1$ & 4 & $5 \cdot 7$ & & & & & $20 \cdot 5^{\star \star}$ & \\
\hline Characters & & & & & & & & & & & & & & & & \\
\hline Human & 17 & $10 \cdot 6$ & 4 & 1.4 & 24 & $100 \cdot 0$ & 129 & 98.0 & 69 & $97 \cdot 1$ & 145 & 99.0 & 46 & 63.0 & $726^{\star \star \star}$ & 0.00 \\
\hline Animated human & 52 & $32 \cdot 3$ & 85 & 29.8 & & 0 & ( & 0 & 2 & 2.9 & 1 & 0.7 & & 0 & $153^{\star \star \star}$ & 0.00 \\
\hline Animated & 47 & $29 \cdot 2$ & 164 & 57.0 & & 0 & 2 & 1.5 & & -0 & ( & 0 & 26 & 35.6 & $271^{\star \star \star}$ & 0.00 \\
\hline Combination & 45 & 28.0 & 32 & 11.2 & & 0 & & 0 & & 0 & ( & 0 & 1 & 1.4 & $117^{\star \star \star}$ & 0.00 \\
\hline Gender & & & & & & & & & & & & & & & & \\
\hline Male & 50 & 31.1 & 137 & $48 \cdot 0$ & 5 & $20 \cdot 8$ & 57 & 43.8 & 14 & $20 \cdot 0$ & 69 & $47 \cdot 3$ & 69 & 94.5 & $111^{\star \star \star}$ & 0.00 \\
\hline Female & 7 & 4.3 & 43 & $15 \cdot 3$ & & 0 & 25 & $19 \cdot 2$ & 14 & $20 \cdot 0$ & 34 & 23.3 & & 0 & $50 \cdot 3^{\star \star \star}$ & 0.00 \\
\hline Combined & 104 & 64.0 & 101 & 35.0 & 19 & 79.0 & 48 & 36.9 & 42 & 60.0 & 43 & 29.5 & 4 & 5.5 & $113^{\star \star \star}$ & 0.59 \\
\hline Major & 151 & 93.0 & 246 & 87.0 & 22 & 91.0 & 106 & 81.0 & 66 & 94.3 & 118 & $80 \cdot 0$ & 64 & 100.0 & $51 \cdot 0^{\star \star \star}$ & 0.00 \\
\hline Goodie & 150 & 93.0 & 265 & 94.0 & 24 & 100.0 & 119 & 91.0 & 68 & 97.1 & 144 & 99.0 & 73 & 100.0 & 20.5 & 0.11 \\
\hline Age & & & & & & & & & & & & & & & & \\
\hline Child & 45 & 39.5 & 19 & $15 \cdot 2$ & 4 & $16 \cdot 7$ & 14 & 10.9 & 4 & $5 \cdot 7$ & 2 & 1.4 & 5 & $10 \cdot 6$ & $85 \cdot 8^{\star \star \star}$ & 0.00 \\
\hline Teen & & 0 & 29 & 23.2 & & 0 & 6 & 4.7 & & 0 & 119 & $81 \cdot 0$ & 2 & 4.3 & $369^{\star \star \star}$ & 0.00 \\
\hline Adult & 41 & 36.0 & 63 & 50.4 & 9 & 37.5 & 77 & 59.7 & 62 & 88.6 & 14 & $9 \cdot 6$ & $3 \overline{7}$ & 78.7 & $166^{\star \star \star}$ & 0.00 \\
\hline Combined & 28 & $24 \cdot 6$ & 14 & 11.2 & 11 & 45.8 & 32 & 24.8 & 4 & 5.7 & 11 & 7.5 & 3 & 6.4 & $49 \cdot 1^{\star \star \star}$ & 0.00 \\
\hline Ethnicity & & & & & & & & & & & & & & & & \\
\hline Caucasian & 91 & 79.8 & 101 & 87.0 & 11 & $45 \cdot 8$ & 120 & 93.0 & 70 & $100 \cdot 0$ & 133 & 91.0 & 45 & 95.7 & $66 \cdot 9^{\star \star \star}$ & 0.00 \\
\hline African & ( & 0 & 5 & 4.3 & & 0 & 2 & 1.6 & & 0 & 1 & 0.7 & 2 & 4.3 & $12 \cdot 2$ & 0.09 \\
\hline Asian & 1 & 0.9 & 1 & 0.9 & & 0 & ( & 0 & & 0 & & 0 & & 0 & 3.65 & 0.82 \\
\hline Hispanic & ( & 0 & ( & 0 & & 0 & ( & 0 & & 0 & ( & 0 & & 0 & & \\
\hline Combined & 22 & 19.3 & 8 & 7.0 & 13 & 54.2 & 7 & 5.4 & & 0 & 12 & $8 \cdot 2$ & & 0 & $83 \cdot 7^{\star \star \star}$ & 0.00 \\
\hline Weight status & & & & & & & & & & & & & & & & \\
\hline Non-overweight & 103 & $90 \cdot 0$ & 134 & 93.0 & 24 & $100 \cdot 0$ & 117 & $90 \cdot 0$ & 66 & $94 \cdot 3$ & 135 & 92.0 & 33 & $70 \cdot 2$ & $29 \cdot 5^{\star \star \star}$ & 0.00 \\
\hline Overweight & 1 & 0.9 & 9 & $6 \cdot 3$ & & 0 & 2 & 1.5 & 2 & 2.9 & 7 & 4.8 & 11 & 23.4 & $45 \cdot 5^{\star \star \star}$ & 0.00 \\
\hline Combined & 10 & 8.8 & ( & 0 & & 0 & 11 & 8.5 & 2 & $2 \cdot 9$ & 4 & $2 \cdot 7$ & 3 & $2 \cdot 6$ & $19 \cdot 6^{\star \star}$ & 0.006 \\
\hline
\end{tabular}

Values given are frequencies and total relative percentages.

Level of significance as: ${ }^{\star} P<0.05$, ${ }^{\star \star} P<0.01$, ${ }^{\star \star \star} P<0.001$.

unspecified beverage type across most genres. Of those specifically shown, there were similar proportions of each of the main beverage types across the various genres, apart from tween programming which displayed a very high proportion of sugar-sweetened beverages.

When cue usage was analysed on the basis of programme genre, the results were largely similar across the genres but a few differences were noted. As expected, quiz programming showed a high proportion of cues with a non-food context. Films and tween programming most commonly involved an act of ingestion. For the majority of genres food and beverage cues were most commonly associated in a setting outside the home, with only tween programming showing a high proportion of food and beverage cues within the home. Previous studies have also shown that most cues are located in a setting outside the home ${ }^{(26)}$.
Throughout the various genres, male characters were most prominent while, as expected, there was a high of proportion of teenage characters in tween programming. Similar proportions of overweight characters were noted through most genres apart from an increased proportion in young persons' programming and no overweight characters within child-specific programmes. We have previously shown that food and beverage cues were most commonly depicted with human characters, and more likely to be white, male and adult in nature ${ }^{(23)}$, in line with previous studies ${ }^{(26)}$. However, the current study was analysed on the basis of genre and as a result certain character types were more prominent in various genres, so it is difficult to make any conclusions regarding characters with this element of bias within the genres.

For each genre apart from quiz programming, the most common motivating factors were celebratory/social and hunger/thirst while there were low proportions of cues 
Table 3 Comparison of motivating factors and outcome in food and beverage cues, by programme genre, in a content analysis of five weekdays of children-specific television broadcasting on both UK (BBC) and Irish (RTE) television channels ( $82.5 \mathrm{~h}$ in total), July 2010 and October 2010

\begin{tabular}{|c|c|c|c|c|c|c|c|c|c|c|c|c|c|c|c|c|}
\hline & \multicolumn{2}{|c|}{$\begin{array}{c}\text { Animated } \\
(n \text { 206) }\end{array}$} & \multicolumn{2}{|c|}{$\begin{array}{l}\text { Cartoon } \\
(n \text { 356) }\end{array}$} & \multicolumn{2}{|c|}{$\begin{array}{l}\text { Child } \\
(n \text { 40) }\end{array}$} & \multicolumn{2}{|c|}{$\begin{array}{l}\text { Film } \\
(n \text { 146) }\end{array}$} & \multicolumn{2}{|c|}{$\begin{array}{l}\text { Quiz } \\
\text { (n 137) }\end{array}$} & \multicolumn{2}{|c|}{$\begin{array}{l}\text { Tween } \\
(n 152)\end{array}$} & \multicolumn{2}{|c|}{$\begin{array}{l}\text { Young person } \\
\text { (n 114) }\end{array}$} & \multirow[b]{2}{*}{$\chi^{2}$} & \multirow[b]{2}{*}{$P$} \\
\hline & $n$ & $\%$ & $n$ & $\%$ & $n$ & $\%$ & $n$ & $\%$ & $n$ & $\%$ & $n$ & $\%$ & $n$ & $\%$ & & \\
\hline \multicolumn{17}{|l|}{ Motivation } \\
\hline Celebratory/social & 58 & 33.5 & 58 & $19 \cdot 9$ & 17 & $54 \cdot 8$ & 41 & $31 \cdot 8$ & 6 & $8 \cdot 6$ & 41 & $28 \cdot 3$ & 9 & $12 \cdot 3$ & $72 \cdot 1^{\star \star \star}$ & 0.00 \\
\hline Hunger/thirst & 34 & 18.5 & 95 & $32 \cdot 6$ & 18 & 58.1 & 35 & $27 \cdot 1$ & 8 & 11.4 & 23 & $15 \cdot 9$ & \multirow{2}{*}{\multicolumn{2}{|c|}{$0^{20 \cdot 0}$}} & $62 \cdot 1^{\star \star \star}$ & 0.0 \\
\hline Upset & \multicolumn{2}{|c|}{$0^{10.0}$} & \multicolumn{2}{|c|}{0} & \multicolumn{2}{|c|}{$0^{30.1}$} & 4 & $3 \cdot 1$ & \multicolumn{2}{|c|}{0} & \multicolumn{2}{|c|}{0} & & & $24 \cdot 8^{\star \star}$ & 0.001 \\
\hline Excitement & 7 & 4.0 & 7 & $2 \cdot 4$ & & 0 & 3 & $2 \cdot 3$ & & ) & 3 & $2 \cdot 1$ & 5 & $6 \cdot 8$ & $16 \cdot 4^{\star}$ & 0.02 \\
\hline Weight/diet & \multicolumn{2}{|c|}{0} & \multicolumn{2}{|c|}{0} & & 0 & \multicolumn{2}{|c|}{0} & \multicolumn{2}{|c|}{0} & \multicolumn{2}{|c|}{0} & \multicolumn{2}{|c|}{0} & & \\
\hline Reward & 6 & 3.5 & 12 & $4 \cdot 1$ & \multicolumn{2}{|r|}{0} & 3 & $2 \cdot 3$ & 8 & 11.4 & 7 & 4.8 & \multicolumn{2}{|r|}{6.8} & 5.54 & 0.59 \\
\hline Punishment & \multicolumn{2}{|c|}{0} & 5 & 1.7 & & 0 & 2 & 1.5 & \multicolumn{2}{|c|}{0} & 4 & $2 \cdot 8$ & \multicolumn{2}{|c|}{0} & 9.01 & 0.25 \\
\hline Health-related & \multicolumn{2}{|c|}{0} & 4 & 1.4 & \multicolumn{2}{|r|}{0} & \multicolumn{2}{|c|}{0} & 12 & $17 \cdot 1$ & \multicolumn{2}{|c|}{0} & 4 & 5.5 & $49 \cdot 1^{\star \star \star}$ & 0.0 \\
\hline Other & 31 & $17 \cdot 9$ & 69 & $23 \cdot 7$ & 2 & 6.5 & 4 & 3.0 & 14 & $20 \cdot 0$ & 13 & $9 \cdot 0$ & 25 & $34 \cdot 2$ & $54 \cdot 1^{\star \star \star}$ & 0.0 \\
\hline Unknown & 48 & $27 \cdot 7$ & 54 & $18 \cdot 6$ & 5 & $16 \cdot 1$ & 43 & $33 \cdot 3$ & 26 & $37 \cdot 1$ & 57 & $39 \cdot 3$ & 15 & 20.5 & $32 \cdot 3^{\star \star \star}$ & 0.0 \\
\hline Positive (\%) & & 8.0 & & 5.3 & & 0.5 & & 4.8 & & 8.9 & & 34.5 & & 4.4 & & \\
\hline Negative (\%) & & 0.0 & & 1.6 & & 0.0 & & 4.4 & & 0.0 & & $2 \cdot 7$ & & 0 & & \\
\hline Neutral (\%) & & 8.5 & & 2.5 & & $2 \cdot 9$ & & 5.6 & & 7.0 & & 5.5 & & 4.4 & & \\
\hline Outcome & & & & & & & & & & & & & & & & \\
\hline Positive & 70 & $40 \cdot 5$ & 89 & 30.5 & 15 & $48 \cdot 4$ & 39 & 29.8 & 24 & $34 \cdot 3$ & 42 & $28 \cdot 8$ & 20 & $27 \cdot 4$ & 11.9 & 0.10 \\
\hline Negative & 33 & $19 \cdot 1$ & 72 & $24 \cdot 7$ & & 0 & 22 & 48.4 & 6 & 8.6 & 37 & $25 \cdot 3$ & 12 & $16 \cdot 4$ & $21 \cdot 9^{\star \star}$ & 0.003 \\
\hline Neutral & 70 & 40.5 & 131 & $36 \cdot 0$ & 16 & 51.6 & 70 & 53.4 & 40 & $57 \cdot 1$ & 67 & $45 \cdot 9$ & 41 & $56 \cdot 2$ & $12 \cdot 4$ & 0.09 \\
\hline
\end{tabular}

Values given are frequencies and total relative percentages.

Level of significance: ${ }^{\star} P<0.05,{ }^{\star \star} P<0.01,{ }^{\star \star \star} P<0.001$.

based on health, reward or punishment motivating factors. Thus, motivating factors were largely independent of genre type, and this is consistent with previous studies which demonstrated that cues were most likely to be social or celebratory motivated ${ }^{(26)}$. The majority of genres demonstrated a low proportion of negative outcomes. Similar results were seen for cue motivating factors and outcome between the various genres. Thus the ingestion of fatty and sugary foods and sugar-sweetened beverages frequently was portrayed positively during the children's programming in the present study. In addition, greater portrayal and ingestion of EDNP foods allows for greater social acceptance. Again, this is further enhanced and strengthened by the frequent associations with celebratory and social motivations, suggesting positive portrayal motives.

A common theme throughout the present study on genre was the more frequent association between tween programming and certain food-related products and behaviours. Tween programming was found to portray the highest proportion of fast foods and convenience foods, and a very high proportion of sugar-sweetened beverages. Additionally, tween programming most commonly showed an act of ingestion, with a large proportion of such cues taking place within the home. Thus, these programmes, which are specifically made for those of teenage years, are designed with a more frequent portrayal of EDNP foods and beverages, in addition to persuasive elements such as character valuing or excess acts of consumption of such foods, with such frequent portrayals potentially having the potential to influence food and beverage choice within this older child and teenage age group.
The precise extent to which such increased exposure to EDNP foods and beverages in children-specific programming leads to increased consumption is as yet not fully determined. As previously highlighted, strong evidence exists that television and in particular advertising influences the food and beverage preferences, purchase requests, beliefs and short-term consumption of children aged between 2 and 11 years but there is insufficient evidence about their influence in those aged between 12 and 18 years $^{(3)}$. Additionally, there is moderate evidence that television advertising influences the usual dietary intake of younger children but weak evidence that it influences the usual dietary intake of older children or teens ${ }^{(3)}$. In the current study there were high proportions of fast foods and convenience foods seen within tween programming and high proportions of sweet snacks and sweets/candy-related cues in animated, cartoon and children-specific genre programmes. As these programme types are aimed primarily at different age groups they may have the potential to differentially influence the choice and preference for specific food types, in particular among viewers of a younger age. This influence of food and beverage portrayal on the basis of age is an exciting area of further research.

Previous studies have examined the influence of product placement in younger and older age-grouped children, showing that those who had seen the branded clip were more likely to select the branded product while age did not appear to be a mediating factor affecting choice $^{(27)}$. Thus by embedding brands within entertainment, these strategies exert a powerful implicit influence on children's memory and choices, typically without explicit awareness of advertising exposure ${ }^{(27)}$. The 
prominent appearance of specific products portrayed in a manner that is congruent with the plot can lead to greater brand awareness and positive attitudes towards that product ${ }^{(28)}$. In support of this a number of studies have looked at the impact of other healthrelated behaviours in television and movies, including smoking, suggesting that children who view smoking are more likely to smoke themselves ${ }^{(29,30)}$. Thus, extrapolating such results from advertising would suggest that there is a high probability of increased consumption with such placements within children-specific programming. However, it is accepted that there is a difference in the portrayal of foods and beverages between advertisement and programming, and thus further examining the influence of programming on consumption is an exciting area for further research.

The present study has limitations. Although the data presented herein represent television broadcasts from three seasons ago, there is little subsequent reason or legislation to suggest that broadcast content has changed in the last three seasons. Further, our findings are consistent with previous studies. Furthermore, the content of advertising during children's broadcast programmes was outside the scope of our study. The study was designed to assess the nutritional quality based on the food pyramid, where foods that should be eaten in moderation or are not recommended are found at the pyramid tip ${ }^{(22)}$, and as such does not reflect current guidelines in countries such as the USA where this has been replaced by MyPlate recommendations ${ }^{(31)}$. Finally, the objectives of the study were to examine content of broadcasts, not the effect of this content on children - but this is an area of interesting future research.

Within the UK statutory rules apply to advertisements for high fat, salt and sugar (HFSS) foods on television channels dedicated to children, in or around programmes aimed at children, and in or around programmes that are likely to be of particular appeal to children aged 4-15 years ${ }^{(32)}$. These provisions prevent: (i) marketing to children that uses promotional offers, licensed characters or celebrities in food advertisements other than for fruit and vegetables; (ii) food advertisements that condone or encourage poor nutritional habits or dietary practice; and (iii) food advertisements that encourage pester power or use hard-sell or high-pressure techniques. In a similar move, recent new broadcasting regulations issued by the Broadcasting Authority of Ireland will disallow advertisements for HFSS content during children's programming ${ }^{(33)}$. Also, within this new version, foods as deemed HFSS in accordance with nutrient profiling systems developed by the UK Food Standards Agency will require advertisers of food and drink products to provide them with nutrition profile certificates, so that broadcasters can deem whether or not a specific product is a HFSS product ${ }^{(33)}$. However, such moves do not currently cover the content of television programmes. Thus, it may be that these current measures do not go far enough to help prevent coverage and influence of television on the portrayal of food products. Future studies should address the impact on the child of watching television programmes depicting food and beverage intake to help further highlight the need to address food-related activities within the wider setting.

\section{Conclusion}

The present study has shown that eating and drinking are common activities across all children's programming types and that EDNP foods and beverages are especially common. This is frequently associated with positive motivating factors and seldom seen with negative outcomes. Tween programming in particular is associated with a high proportion of unhealthy eating and drinking behaviours which may have implications for food and beverage preferences. In the future such programming should therefore involve the active inclusion of more frequent and positively associated connotations with healthy foods and behaviours. So in addition to attempting to restrict the amount of time spent watching television programming, it is also important to change the messages being portrayed regarding food and eating habits in the programmes that are viewed.

\section{Acknowledgements}

Financial support: This research received no specific grant from any funding agency in the public, commercial or not-for-profit sectors. Conflict of interest: None. Authorship: P.S. designed data collection, scored recordings, analysed data, and drafted and revised the paper. $\mathrm{He}$ is guarantor. O.R. designed data collection, scored recordings and analysed data. A.M. recorded data, designed data collection, analysed data and revised the paper. M.H. wrote the statistical analysis plan, monitored data collection and revised the draft paper. J.S. wrote the statistical analysis plan and monitored data collection. D.L. initiated the project and revised the paper. W.C. initiated the project and revised the paper. C.D. initiated the project, monitored data collection and revised the paper. C.S.O. initiated the project, designed data collection, implemented the trial, monitored data collection, analysed the data and revised the paper. Ethics of buman subject participation: Ethical approval was not required.

\section{References}

1. Reilly JJ (2006) Obesity in childhood and adolescence: evidence based clinical and public health perspectives. Postgrad Med J 82, 429-437.

2. Saunders T (2011) Potential contributors to the Canadian pediatric obesity epidemic. ISRN Pediatr 2011, 917684. 
3. McGinnis MJ, Gootman JA \& Kraak VI (2006) Health, diet, and eating patterns of children and youth. Food Marketing to Children and Youth: Threat or Opportunity?, pp. 39-90. Washington, DC: National Academies Press.

4. Gortmaker SL, Must A, Sobol AM et al. (1996) Television viewing as a cause of increasing obesity among children in the United States, 1986-1990. Arch Pediatr Adolesc Med 150, 356-362.

5. Kaiser Family Foundation (2004) The role of media in childhood obesity. http://www.kff.org/entmedia/ entmedia022404pkg.cfm (accessed October 2014).

6. Strasburger VC (2006) Children, adolescents, and advertising. Pediatrics 118, 2563-2569.

7. Powell LM, Szczypka G, Chaloupka FJ et al. (2007) Nutritional content of television food advertisements seen by children and adolescents in the United States. Pediatrics 120, 576-583.

8. Ofcom (2014) Communications Report. http://stakeholders. ofcom.org.uk/market-data-research/market-data/communica tions-market-reports/cmr14/uk/ (accessed December 2014).

9. Harrison K \& Marske AL (2005) Nutritional content of foods advertised during the television programs children watch most. Am J Public Health 95, 1568-1574.

10. Henderson VR \& Kelly B (2005) Food advertising in the age of obesity: content analysis of food advertising on general market and African American television. J Nutr Educ Behav 37, 191-196.

11. Story M \& French S (2004) Food advertising and marketing directed at children and adolescents in the US. Int J Behav Nutr Phys Act 1, 3.

12. Halford JC, Gillespie J, Brown V et al. (2004) Effect of television advertisements for foods on food consumption in children. Appetite 42, 221-225.

13. Halford JC, Boyland EJ, Hughes GM et al. (2008) Beyondbrand effect of television food advertisements on food choice in children: the effects of weight status. Public Health Nutr 11, 897-904.

14. Taras HL \& Gage M (1995) Advertised foods on children's television. Arch Pediatr Adolesc Med 149, 649-652.

15. Lewis MK \& Hill AJ (1998) Food advertising on British children's television: a content analysis and experimental study with nineyear olds. Int J Obes Related Metab Disord 22, 206-214.

16. Strachan J \& Pavie-Latour V (2008) Food for thought: shouldn't we actually target food advertising more towards kids and not less? Int J Mark Res 50, 13-27.

17. von Normann K (2009) The impact of lifestyles and food knowledge on the food patterns of German children. Int J Consum Stud 33, 382-391.

18. Elliott CD (2009) Healthy food looks serious: how children interpret packaged food products. Can J Commun 34, 359-380.

19. Atik D \& Ertekin ZO (2013) Children's perception of food and healthy eating: dynamics behind their food preferences. Int J Consum Stud 37, 59-65.
20. Broadcasting Authority of Ireland (2010) Strategy Statement 2011-2013. http://www.bai.ie/wordpress/wp-content/ uploads/BAI_Strategy_201113_v2ENG.pdf (accessed October 2014).

21. British Broadcasting Corporation (2006) BROADCASTING: An Agreement Between Her Majesty's Secretary of State for Culture, Media and Sport and the British Broadcasting Corporation. http://downloads.bbc.co.uk/bbctrust/assets/ files/pdf/about/how_we_govern/agreement.pdf (accessed October 2014).

22. Department of Health (2012) Your guide to healthy eating using the Food Pyramid. http://health.gov.ie/blog/publica tions/your-guide-to-healthy-eating-using-the-food-pyramid/ (accessed March 2015).

23. Scully P, Reid O, Macken A et al. (2014) Food and beverage cues in United Kingdom and Irish children-television programming. Arch Dis Child 99, 979-984.

24. Radnitz C, Byrne S, Goldman R et al. (2009) Food cues in children's television programs. Appetite 52, 230-233.

25. Boynton-Jarrett R, Thomas TN, Peterson KE et al. (2003) Impact of television viewing patterns on fruit and vegetable consumption among adolescents. Pediatrics 112, $1321-1326$

26. Greenberg BS, Rosaen SF, Worrell TR et al. (2009) A portrait of food and drink in commercial TV series. Health Commun 24, 295-303.

27. Auty S \& Lewis C (2004) Exploring children's choice: the reminder effect of product placement. Psychol Mark 21, 697-713.

28. Russell CA (2002) Investigating the effectiveness of product placement in television shows: the role of modality and plot connection congruence on brand memory and attitude. J Consum Res 29, 306-319.

29. Sargent JD, Beach ML, Adachi-Mejia AM et al. (2005) Exposure to movie smoking: its relation to smoking initiation among US adolescents. Pediatrics 116, 1183-1191.

30. Dalton MA, Sargent JD, Beach ML et al. (2003) Effect of viewing smoking in movies on adolescent smoking initiation: a cohort study. Lancet 362, 281-285.

31. US Department of Agriculture (2012) ChooseMyPlate. gov. http://www.choosemyplate.gov/downloads/Getting StartedWithMyPlate.pdf (accessed March 2015).

32. Committees of Advertising Practice (2010) UK Code of Broadcast Advertising (BCAP Code). http://www.cap.org uk/Advertising-Codes/Broadcast.aspx (accessed December 2014).

33. Broadcasting Authority of Ireland (2012) BAI signals new rules to govern advertising of food and drink in children's advertising. http://www.bai.ie/?p=2792 (accessed December 2014). 